\title{
Fusion Analytics: A Data Integration System for Public Health and Medical Disaster Response Decision Support
}

\author{
Dina B. Passman* \\ ASPR/OPEO, HHS, Washington, DC, USA
}

\section{Objective}

The objective of this demonstration is to show conference attendees how they can integrate, analyze, and visualize diverse data type data from across a variety of systems by leveraging an off-the-shelf enterprise business intelligence (EBI) solution to support decisionmaking in disasters.

\section{Introduction}

Fusion Analytics is the data integration system developed by the Fusion Cell at the U.S. Department of Health and Human Services (HHS), Office of the Assistant Secretary for Preparedness and Response (ASPR). Fusion Analytics meaningfully augments traditional public and population health surveillance reporting by providing webbased data analysis and visualization tools.

\section{Methods}

Fusion Analytics serves as a one-stop-shop for the web-based data visualizations of multiple real-time data sources within ASPR. The 24-7 web availability makes it an ideal analytic tool for situational awareness and response allowing stakeholders to access the portal from any internet-enabled device without installing any software. The Fusion Analytics data integration system was built using off-the-shelf EBI software. Fusion Analytics leverages the full power of statistical analysis software and delivers reports to users in a secure web-based environment. Fusion Analytics provides an example of how public health staff can develop and deploy a robust public health informat- ics solution using an off-the shelf product and with limited development funding. It also provides the unique example of a public health information system that combines patient data for traditional disease surveillance with manpower and resource data to provide overall decision support for federal public health and medical disaster response operations.

\section{Conclusions}

We are currently in a unique position within public health. One the one hand, we have been gaining greater and greater access to electronic data of all kinds over the last few years. On the other, we are working in a time of reduced government spending to support leveraging this data for decision support with robust analytics and visualizations. Fusion Analytics provides an opportunity for attendees to see how various types of data are integrated into a single application for population health decision support. It also can provide them with ideas of how they can use their own staff to create analyses and reports that support their public health activities.

\section{Keywords}

Situational awareness; Public health informatics; Disaster response

*Dina B. Passman

E-mail: dina.passman@hhs.gov 